\title{
The Relation Between ADHD and Cognitive Profiles of Children with NF1
}

\author{
Deborah Potvin $^{1} \cdot$ Kristina K. Hardy ${ }^{1}$ Karin S. Walsh ${ }^{1}$
}

Received: 25 February 2015 /Revised: 3 September 2015 / Accepted: 29 September 2015 /Published online: 7 October 2015

(C) American Academy of Pediatric Neuropsychology 2015

\begin{abstract}
Despite well-accepted findings of a "downward shift" in intellectual functioning in children with neurofibromatosis type 1 (NF1), research has not examined the contribution of the individual indices in this lower performance on intelligence (IQ) measures. Although $30-50 \%$ of children with NF1 meet criteria for a diagnosis of attention deficit/ hyperactivity disorder (ADHD), research has not established the relation between ADHD and intellectual profiles in these children. To clarify these issues, this study examined the Wechsler Intelligence Scale for Children, Fourth Edition (WISC-IV) profiles of children with NF1, with and without comorbid ADHD, using a comparison group of children with developmental ADHD. We found that rather than an overall lowering of IQ, children with NF1 demonstrated an uneven intellectual profile. While verbal abilities were generally preserved, nonverbal, working memory, and processing speed abilities were lower than the population norms. Children with NF1 and comorbid ADHD showed significantly lower performance on tests of working memory than children with NF1 without ADHD or children with developmental ADHD. These results suggest that global intellect is inadequate in describing the cognitive phenotype in children with NF1 and that in children with NF1 comorbid ADHD may manifest as additional cognitive burden with respect to working memory.
\end{abstract}

Keywords Neurofibromatosis type $1(\mathrm{NF} 1) \cdot$ Attention deficit/hyperactivity disorder (ADHD) - Wechsler Intelligence Scale for Children, Fourth Edition (WISC-IV) · Cognitive profile $\cdot$ IQ profile

Karin S. Walsh

KWalsh@childrensnational.org

1 Children National Medical Center, Washington, DC, USA
Neurofibromatosis type 1 (NF1) is an autosomal dominant disorder that affects both the peripheral and central nervous systems. Diagnosis of NF1 is made upon observation of café au lait macules, freckling, Lisch nodules, neurofibromas, optic gliomas, or osseous lesions (Ferner et al. 2007; Gutmann et al. 1997). However, the disorder is highly variable in terms of clinical presentation, with only a minority of children experiencing significant physical complications (Huson et al. 1989). In contrast, cognitive dysfunction is wide ranging, affecting approximately $80 \%$ of children with NF1 in one capacity or another (Hyman et al. 2005; Hyman et al. 2006).

Investigations of the neuropsychological profiles of children with NF1 have consistently documented a general "downward shift" in overall IQ (Ferner et al. 1996), with the mean intellectual functioning of children with NF1 in the low end of the average to low average range (Levine et al. 2006; North 1998, 2000). Beyond intellect, studies have found specific deficits in visual perception, most consistently on the Benton Judgment of Line Orientation (JLO) task (Rowbotham et al. 2009; Hyman et al. 2005; Schrimsher et al. 2003; Benton et al. 1983), along with more generalized difficulties in the areas of language development (Hyman et al. 2005), executive functioning (Templer et al. 2012; Roy et al. 2010; North et al. 2002), and attention (Templer et al. 2012; North et al. 2002). Understanding of these deficits has helped to guide intervention trials and clinical practice with the NF1 population, shaping assessment and intervention practices.

Despite some evidence of greater performance on verbal intellectual (VIQ) subtests compared with nonverbal or performance intellectual subtests (PIQ) (Lidzba et al. 2012), a specific IQ profile associated with NF1 has not been established. In contrast, specific intellectual profiles have been established in a number of developmental disorders and have been linked to diagnostic subtype (Thaler et al. 2012), social 
functioning (Oliveras-Rentas et al. 2011; Black et al. 2009), and behavioral functioning (Thaler et al. 2012). For example, research has shown specific patterns of intellectual functions in children with developmental attention deficit/hyperactivity disorder (ADHD). Similar to children with NF1, children with developmental ADHD demonstrate a general lowering of global intellect (Mayes and Calhoun 2006; Bridgett and Walker 2006; Frazier et al. 2004), but a profile has emerged, characterized by specific deficits in the working memory and processing speed indices relative to verbal and nonverbal reasoning indices (Mayes and Calhoun 2006). This profile captures the executive function deficits that are characteristic of ADHD

ADHD is highly prevalent within the NF1 population, with 31 to $50 \%$ of children meeting diagnostic criteria for ADHD (Payne et al. 2011; Pride et al. 2012; Hyman et al. 2005; Rosser and Packer 2003). This translates to a 3- to 12-fold increase risk of ADHD in children with NF1 when compared to the $5 \%$ prevalence in children in the general population (APA 2013). Thus, in addition to providing a model for the ways in which understanding the characteristic intellectual profile of a developmental disorder can shape research and treatment, the high rate of ADHD within the NF1 population also raises the question of what role comorbid ADHD may play in the cognitive profile of NF1.

Recent research has begun to investigate whether children with NF1 and comorbid ADHD represent a distinct subgroup within NF1, with qualitatively different areas of cognitive deficit, or whether the cognitive deficits associated with NF1 are merely exacerbated by comorbid ADHD. Several recent studies have found evidence that comorbid ADHD is associated with additional cognitive burden in areas already affected by NF1, including language (Pride et al. 2012), strategic planning (Roy et al. 2010), and academic achievement (Pride et al. 2012). However, findings on the relation between ADHD and the cognitive profile of NF1 have been variable. For example, one study suggested that children with NF1 and comorbid ADHD do not show additional deficits in executive functioning, beyond those already associated with NF1 (Payne et al. 2012).

Alternatively, a study examining performance on the Wechsler Intelligence Scale for Children, German version, found that the presence of comorbid ADHD had a global negative impact on intellectual functioning and that children with NF1 without comorbid ADHD were not distinguishable from the general population (Lidzba et al. 2012). These findings, though preliminary, suggest that the cognitive deficits commonly associated with NF1 may, in fact, be confined to the subgroup of the NF1 population with comorbid ADHD. Despite the striking nature of these findings, Lidza et al. were not able to clarify the impact of comorbid ADHD on the intellectual profile of children with NF1, due to the lack of a comparison group with developmental ADHD. Thus, a comparison with children with developmental ADHD is necessary to dissect the convergence or divergence of the two disorders and the relation between ADHD in the cognitive profile of NF1.

We propose to further investigate these questions by examining the intellectual profiles of children with NF1, with and without comorbid ADHD (NF1 and NF1+ADHD). In order to better clarify the role of ADHD in NF1, we include a comparison group of children with developmental ADHD. Based on previous research on the intellectual profiles associated with NF1, we hypothesize that all children with NF1 will show relative deficits on nonverbal/performance subtests when compared to verbal subtests. We also hypothesize that consistent with research on other neurodevelopmental disorders, children with NF1 and children with developmental ADHD will show a pattern of deficits in working memory and processing speed in comparison to verbal and nonverbal domains. Finally, we hypothesize that children with NF1+ ADHD will exhibit greater relative deficits in these two areas, associated with the dual burden of the comorbid diagnoses.

\section{Methods}

\section{Participants and Procedures}

Following approval from the Institutional Review Board (IRB) of Children's National Medical Center, cognitive and demographic data were abstracted from the medical records of children with NF1 and/or ADHD who had been evaluated in the Division of Neuropsychology in a metropolitan academic medical children's hospital. Participants were eligible if they were diagnosed with either NF1, ADHD, or both, aged 616 years, and had completed the Wechsler Intelligence Scale for Children, Fourth Edition. NF1 diagnosis was confirmed by medical evaluation and participants met NIH diagnostic criteria for the disorder (NIH 1987). As per best-practice guidelines, ADHD diagnosis was established with a clinical interview, parent and teacher questionnaire ratings of ADHD symptoms, and a comprehensive review of the participant's developmental and medical history. Within the NF1 group, a small portion of the participants $(n=7)$ had reports that did not comment directly on the presence or absence of comorbid ADHD. In these cases, participants were classified as having comorbid ADHD if ADHD rating scales from parents and quantitative assessment of attention and/or impulse control met the diagnostic criteria for ADHD $(n=2)$ or if they were actively taking a stimulant medication $(n=1)$. One participant with NF1, whose clinical report specifically indicated no diagnosis of ADHD but who was taking a stimulant medication, was excluded from the study. Our sample included 27 children with NF1 only (mean age $=10.93$ years), 23 children with NF1 + ADHD (mean age $=8.78$ years), and 23 children with neurodevelopmental ADHD (mean age $=10.26$ years). 


\section{Measures}

Wechsler Intelligence Scale, Fourth Edition (WISC-IV; Wechsler 2003) All participants completed assessment with the WISC-IV, as part of a comprehensive neuropsychological battery. The WISC-IV includes ten subtests, which comprise four index scores (Verbal Comprehension Index, Perceptual Reasoning Index, Working Memory Index, and Processing Speed Index). The Full Scale IQ (FSIQ) is a composite of all four indices. The Perceptual Reasoning Index (PRI) subtests, which were examined individually within the study, include measures of both visual-spatial (block design) and nonverbal reasoning (matrix reasoning, picture concepts).

\section{ADHD Rating Scale-IV (ADHD-RS; DuPaul et al. 1998)} This is a symptom rating questionnaire, which consists of 18 items reflecting the core symptoms of ADHD (9 inattentive type [In] and 9 hyperactive/impulsive type [HI]) as defined by the DSM-IV (APA 2000). The measure employs a Likert response scale ranging from 0 (symptom "never" occurs) to 3 (symptom "very often" occurs). Results from the home/parent version of this measure were used, along with interview, observation, and neuropsychological test results, to determine the presence or absence of ADHD.

\section{Statistical Analyses}

Following descriptive analyses and a MANOVA to ensure that stimulant medication did not significantly alter the profiles of children in either ADHD group, we used repeated measures multivariate analysis of covariance (MANCOVA) to examine the intellectual profiles of the NF1, NF1+ADHD, and developmental ADHD samples across the four indices of the WISC-IV. Subtest-level analyses were approached with MANCOVAs to better understand the source of any significant differences between the groups. Given historical findings of specific deficits in visual-spatial functions, we included a subtest-level analysis of the Perceptual Reasoning Index, which includes measures of visual-spatial and nonverbal reasoning. Post hoc comparisons between the samples were performed using Tukey-Kramer, and interaction effects were examined using analysis of covariance (ANCOVA). For a more in-depth examination of the prevalence of deficits experienced by the different clinical groups, we calculated the percentage of children demonstrating impairment in each of the four WISC-IV indices, which was defined as performance $\geq 1$ standard deviation below the population mean. We then employed chi-squared statistics and odds ratios to examine rates of impairment across groups.

\section{Results}

\section{Descriptive Findings}

Descriptive statistics for the three groups (NF1, NF1+ADHD, and developmental ADHD) are presented in Table 1. The three groups did not differ in terms of gender, use of special education services, or overall IQ, but children with NF1+ ADHD were significantly younger than the other two groups (see Table 1). For both the NF1-only group and the NF1+ ADHD group, Full Scale IQ (FSIQ) and performance on the Perceptual Reasoning (PRI), Working Memory (WMI), and Processing Speed (PSI) indices were all significantly below the population mean of 100 , while performance on the Verbal Comprehension Index (VCI) was consistent with the population mean. For the developmental ADHD group, FSIQ, along with performance on the WMI and PSI, was significantly below the population mean. Given the significant age difference between groups, age at evaluation was included as a covariate in analyses which included all three groups (NF1, NF1+ADHD, and developmental ADHD).

\section{Medication Effects}

The use of stimulant medication did not differ significantly between the ADHD and NF1+ADHD group $\left(X^{2}(1, N=\right.$ $46)=.383, p=.536$ ). In addition, given the potential confounding cognitive benefits of being on a stimulant medication during evaluation, we examined the relationship between stimulant medication and intellectual profiles and did not find a statistically significant relationship $(F(1,43)=.136 p=.714$, $\left.\eta_{p}{ }^{2}=.003\right)$.

\section{Within-Group Findings}

The intellectual profiles of the combined NF1 group and developmental ADHD group are presented in Fig. 1. There were significant discrepancies between index scores for the NF1 group $\left(F(3,147)=7.845, p=.000, \eta_{p}{ }^{2}=.138\right)$, with performance on PRI, PSI, and WMI significantly lower than performance on VCI, which was within the average range. The profile of the developmental ADHD group also showed significant discrepancies between indices $(F(3,63)=4.191$, $p=.009, \eta_{p}{ }^{2}=.166$ ), with performance on the PSI significantly weaker than performance on the VCI or PRI. The profiles of both groups showed a similar pattern with higher scores on the VCI and PRI compared to the WMI and PSI, which were consistently the lowest for both groups.

\section{Between-Group Findings}

The intellectual profiles of the three groups-NF1, NF1+ ADHD, and developmental ADHD - are presented in Fig. 2. 
Table 1 Sample characteristics $(n=73)$

\begin{tabular}{|c|c|c|c|c|}
\hline & $\begin{array}{l}\text { NF1 } \\
(n=27)\end{array}$ & $\begin{array}{l}\text { NF1 and ADHD } \\
(n=23)\end{array}$ & $\begin{array}{l}\text { ADHD } \\
(n=23)\end{array}$ & \\
\hline Variable & Mean (SD) & Mean (SD) & Mean (SD) & $F(p)$ \\
\hline Age & $10.93(2.87)$ & $8.78(2.21)$ & $10.26(3.35)$ & $F(2,70)=3.614, p=.032$ \\
\hline IQ & $94.78(11.65)^{*}$ & $90.78(13.23)^{* *}$ & $94.83(10.50)^{*}$ & $F(2,70)=.907, p=.408$ \\
\hline Verbal comprehension & $102.59(15.05)$ & $96.91(13.03)$ & $96.23(10.04)$ & \\
\hline Perceptual reasoning & $94.00(11.304)^{* *}$ & $92.09(14.04)^{* *}$ & $100.05(12.47)$ & \\
\hline Working memory & $95.15(10.992)^{*}$ & $88.13(13.14)^{* *}$ & $94.45(12.56)^{*}$ & \\
\hline Processing speed & $89.56(11.03)^{* *}$ & $92.09(14.61)^{* *}$ & $89.91(11.34)^{* *}$ & \\
\hline Variable & $n(\%)$ & $n(\%)$ & $n(\%)$ & $X^{2}$ test \\
\hline Male gender & $12(44)$ & $11(48)$ & $12(52)$ & $\begin{array}{l}X^{2}(2, N=73)=.298 \\
p=.862\end{array}$ \\
\hline Special education services & $9(35)$ & $11(50)$ & $6(29)$ & $\begin{array}{l}X^{2}(2, N=69)=2.27 \\
p=.322\end{array}$ \\
\hline
\end{tabular}

*Differs from the population mean at the $p \leq .05$ level; **differs from the population mean at the $p \leq .01$ level

Repeated-measures MANCOVA results indicated a significant difference in the profiles of the three groups $(F(6,204)=$ $\left.2.867, p=.011, \eta_{p}{ }^{2}=.078\right)$. Specifically, post hoc analysis showed that children with NF1 and comorbid ADHD performed significantly lower on the WMI than either the NF1 only or developmental ADHD group $(F(2,68)=3.571$, $p=.034, \eta_{p}{ }^{2}=.095$ ). A repeated-measures MANCOVA indicated no significant discrepancies within the WMI subtest scores $\left(F(1,68)=1.555, p=.217, \eta_{p}{ }^{2}=.022\right)$, with all groups showing similar patterns of performance across subtests $\left(F(2,68)=574, p=.566, \eta_{p}{ }^{2}=.017\right)$.

\section{Perceptual Reasoning Subtest Findings}

The profiles for the subtests of the PRI are presented in Fig. 3. Consistent with previous research showing specific deficits in visual-spatial processing, children in both of the NF1 groups demonstrated significant discrepancies across the PRI subtest scores $\left(F(2,96)=14.659, p=.000, \eta_{p}{ }^{2}=.234\right)$, with specific weakness on a task of constructional praxis (block design). Although children within the developmental ADHD group

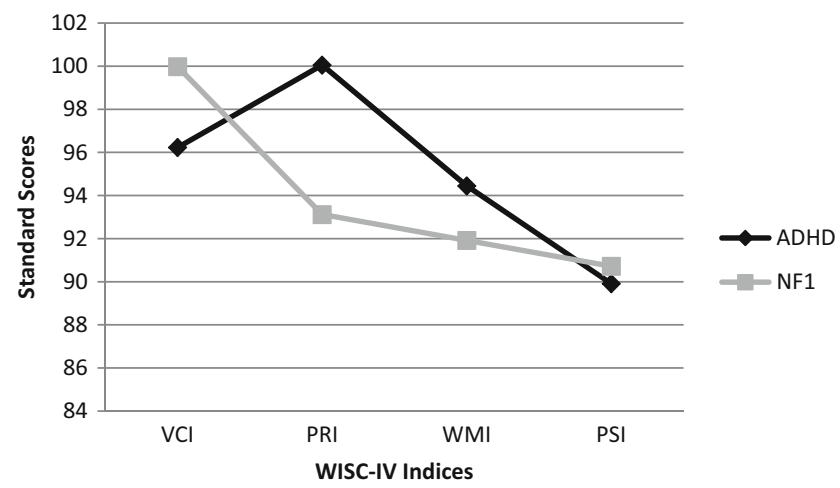

Fig. 1 NF1 and ADHD WISC-IV profile performed higher than children in both of the NF1 groups on all subtests, this difference did not reach statistical significance $\left(F(2.67)=2.376, p=.101, \eta_{p}{ }^{2}=.066\right)$ and all groups showed similar patterns of performance across subtests $(F(4$, $134)=.338, p=.852, \eta_{p}{ }^{2}=.010$ ).

\section{Impairment Levels}

A graph of the percentage of children in each diagnostic group whose performance fell in the impaired range, defined as $\geq 1$ standard deviation below the population norm, is presented in Fig. 4. Consistent with the intellectual profiles, children with NF1+ADHD show higher rates of impairment across all index scores than both other groups, with statistically significant differences in impairment levels for the WMI $\left(X^{2}(2, N=72)=4.589, p=.05\right.$ (one-tailed)). Odds ratios were calculated and showed that children with NF1+ADHD were 2.64 times more likely to be impaired on the WMI than children with NF1 only and 2.15 times more likely than children with developmental ADHD.

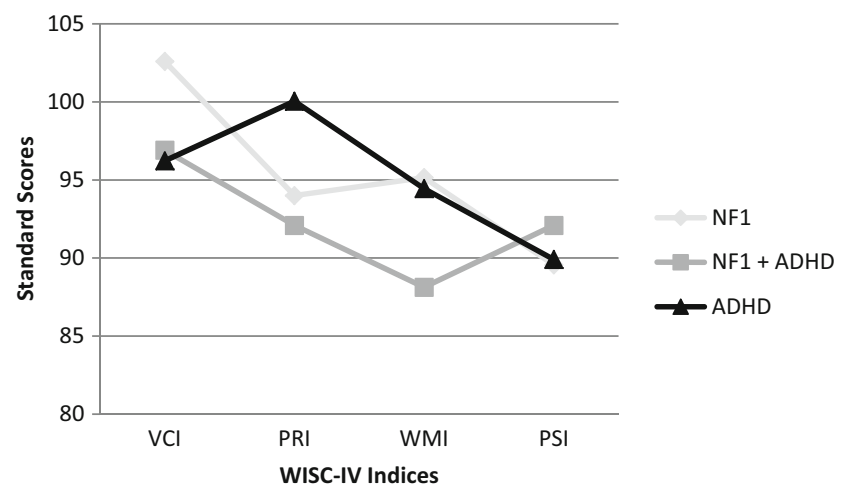

Fig. 2 Comparison of WISC-IV profiles across groups 


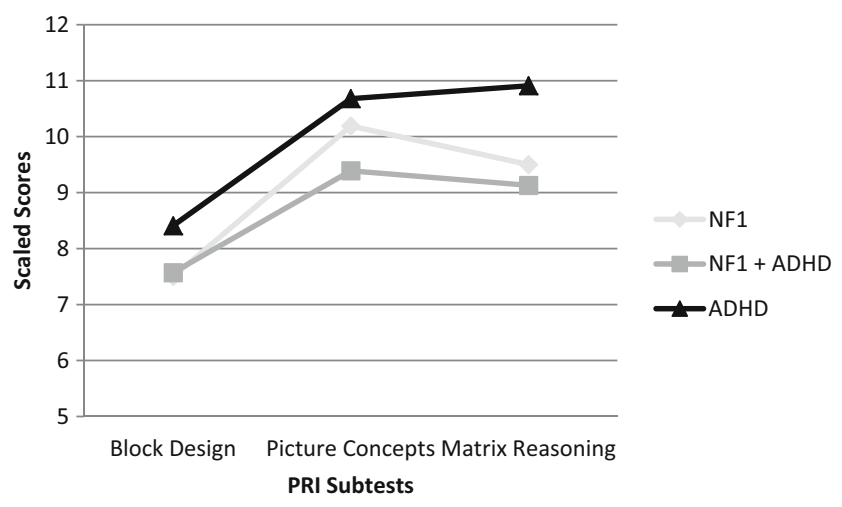

Fig. 3 Comparison of PRI subtests across groups

\section{Discussion}

Consistent with previous research on children with NF1 (Hyman et al. 2005; Rosser and Packer 2003), $45 \%$ of our NF1 group met criteria for a diagnosis of ADHD. Although population studies of developmental ADHD show a clear gender imbalance, with the diagnosis being twice as common in boys (Ullebo et al. 2012; APA 2013), neither the NF1+ ADHD nor the developmental ADHD group showed such a gender imbalance. The relatively equal gender distribution may represent greater sensitivity to symptoms of inattention, which are more common than hyperactivity symptoms in girls (Froehlich et al. 2007) and which are more prevalent, also, within the NF1 population (Pride et al. 2012); however, the ADHD subtype was not consistently documented in the clinical reports and, thus, was not included in this study.

One of the primary aims of this study was to examine the intellectual profile of children with NF1, particularly focusing on whether the well-documented downward shift in IQ represents a global lowering of performance or specific areas of cognitive weakness that served to depress the FSIQ. Consistent with prior research showing a pattern of stronger performance on verbal intellectual measures (Lidzba et al. 2012) and with well-established findings of deficits in

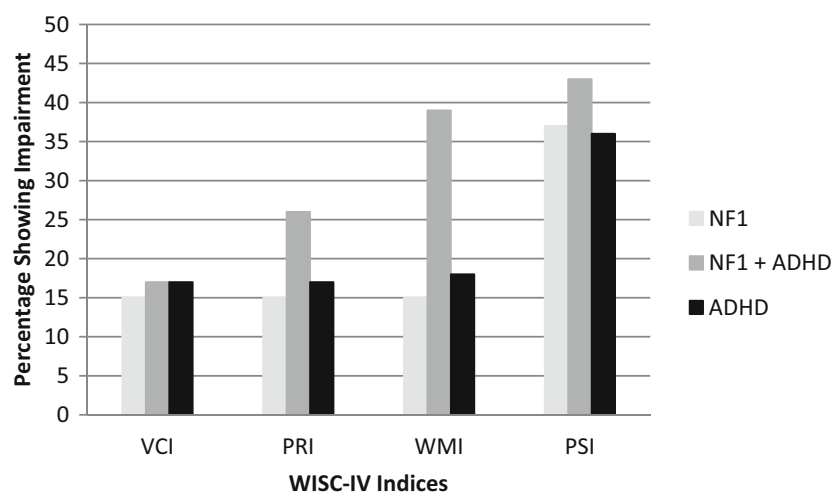

Fig. 4 Percentage showing impairment by group visual-spatial skills (Rowbotham et al. 2009; Hyman et al. 2005; Schrimsher et al. 2003), children with NF1 demonstrated a significant relative strength on verbally mediated tasks, performing at a level just below the population mean, compared to nonverbal/visual-spatial tasks, on which the mean performance was at the low end of the average range.

This intellectual profile shows both broad similarities with profiles of other developmental disabilities and characteristics that may be more specific to NF1. In regard to weaknesses on tasks measuring attentional control, working memory, and cognitive processing and response speed, the NF1 IQ profile is in keeping with research on other developmental disabilities, showing a consistent susceptibility to disruption in attention, working memory, and cognitive efficiency functions (Oliveras-Rentas et al. 2011; Mayes and Calhoun 2006). These types of neuropsychological deficits are linked to disruptions in white matter tracts, which are particularly vulnerable due to their protracted developmental trajectory (e.g., Barnea-Goraly et al. 2005) and are commonly seen in developmental disabilities including NF1, ADHD, and autism spectrum disorders (ASD) (e.g., Lawrence et al. 2013; Weinstein et al. 2011; Cutting et al. 2002). From this perspective, the IQ profile seen in children with NF1 reflects similar vulnerabilities as those observed in other developmental disabilities, such as ADHD and ASD. However, children with NF1 also show a weakness on measures of visual reasoning, a cognitive deficit that is consistent with prior NF1 research and appears to be a unique characteristic of NF1 and in contrast to other developmental disabilities.

This profile contradicts earlier declarations of a generalized downward shift in intellect, instead demonstrating important areas of relative strength and weakness. This finding raises the practical consideration that utilizing global intellectual indices (FSIQ) may not be the most appropriate representation of cognitive functioning in children with NF1, since global indices will underestimate verbal intellectual functioning and overestimate other functions important in academic success, specifically working memory, cognitive efficiency, and visual reasoning. This distinction may be particularly important within the context of making educational determinations about adequate academic progress and the need for special education services.

The other primary goal of this study was to augment recent research on the relation between comorbid ADHD and the cognitive profile of NF1, particularly focusing on whether children with NF1 and ADHD represent a distinct subgroup and whether comorbid ADHD correlates with lowering of all areas of cognitive functioning or only specific aspects, which would suggest a unique relation between ADHD and the cognitive phenotype associated with NF1.

Consistent with previous findings on the relation between ADHD and the intellectual profile of children with NF1 (Lidzba et al. 2012), children with NF1 and comorbid 
ADHD in our sample showed higher levels of impairment in most areas than the children with NF1 or ADHD exclusively. Beyond the impairment levels, the analysis of the intellectual profiles of the three groups-NF1, NF1+ADHD, and developmental ADHD_lends support to a more nuanced picture of cognitive burden associated with the dual diagnoses of NF1 and ADHD. The profile analysis suggested that this comorbidity is associated with impaired performance on tasks of attention and executive functioning (working memory subtests) - core deficits present in developmental ADHD. In contrast, children with NF1 and comorbid ADHD did not show any additional burden on the processing speed subtests but were similar to the levels of impairment in both the developmental ADHD and the NF1 without ADHD groups. Given that performance on the PSI is lower for children with other developmental disabilities, such as ASD (Oliveras-Rentas et al. 2011), these findings are particularly unexpected and warrant further investigation.

Finally, consistent with previous research demonstrating deficits in visual-spatial reasoning in NF1 (Rowbotham et al. 2009; Hyman et al. 2005; Schrimsher et al. 2003), a more focused examination of nonverbal functions found specific deficits in constructional praxis, which relies most heavily on visual-spatial processing, a known area of impairment specific to NF1. In addition, consistent with findings that deficits in visual-spatial processing in NF1 are independent of comorbid ADHD, children with comorbid ADHD did not show significantly lower performance on this subtest than those with NF1 alone.

This study has implications for the evaluation, conceptualization, and treatment of children with cognitive deficits associated with NF1. Our findings highlight the association of specific cognitive limitations in processing speed and working memory with the lower overall IQ levels in children with NF1. This speaks to the importance of considering specific patterns of cognitive strength and weakness in an individual child, rather than basing expectations for academic achievement on global intellectual levels. This will lead to the development of more appropriate and specific educational accommodations and interventions to support specific areas of weakness. School districts that continue to use a discrepancy formula to diagnose a specific learning disability, which bases the diagnosis on unexpected impairments in academic function as compared to the child's IQ, should consider using the General Ability Index (GAI) instead of the FSIQ, as a more appropriate estimate of cognitive ability for such purposes (Raiford et al. 2005). In addition, given the association of comorbid ADHD with greater cognitive impairment in children with NF1, our study also supports early evaluation, diagnosis, and treatment of ADHD in this population.

The findings of our research also speak to the inappropriateness of using global estimates of intelligence in the context of research. This is particularly true with regard to clinical trials in which the primary research questions target response to intervention. Given the relatively stable nature of intellectual development over time, global intellect as a primary outcome would be highly unlikely to be sensitive enough to detect such an effect (Moffitt et al. 1993). Further, as we have shown in this research, utilization of overall intellectual functioning results in missed opportunities for detecting change in specific areas of impairment and in those areas that are known to be more fluid (e.g., attention, working memory, efficiency).

There are some limitations to consider when examining the results of this research. Due to the use of a retrospective clinical sample, participants in this study could not be matched based on demographic features such as age, creating potential confounding variables. In particular, though age at the time of assessment was statistically controlled, the younger age of the $\mathrm{NF} 1+\mathrm{ADHD}$ group raises some concerns about the possible attenuation of ADHD symptoms in children with NF1 as they grow. Likewise, the sample did not allow for the use of a sibling pair-wise design to control for genetic and environmental influences and to clarify the contributions of both factors in the ADHD and NF1 profiles, nor did we have adequate longitudinal data necessary to analyze relationships between cognitive deficits and future academic, social, or functional morbidity. In addition, the use of a sample of children seeking assessment may limit the generalizability of these findings to those in the general NF1 population who are not experiencing functional impacts related to neuropsychological weaknesses. However, the standard of care in our institution is such that functional impairment is not a required criterion for referral for neuropsychological assessment, as the known risks for alterations in cognitive development in this population is adequate for referral and is standard of care. One final limitation of this study was the lack of a clinical judgment on the presence of comorbid ADHD for a small subset of the NF1 sample. Given the almost exclusive use of stimulant medication in children to treat ADHD, medication was used as a proxy for these few children. This may raise concern that medication effects may have impacted performance; however, we did not find an association between stimulant use and intellectual profiles for children with NF1 and comorbid ADHD.

The present study relies on information collected from the WISC-IV, an intelligence test battery that has now been replaced by a newer edition, the WISC-V (Wechsler 2014). While clinically, the use of the WISC-IV is phasing out, this fact does not negate the core findings about the cognitive profiles within these populations. Because of the new structure of the WISC-V, future research is warranted to determine how our findings translate to performance on the WISC-V. An initial review suggests that these findings should translate very well and that the WISC-V, in separating visual-spatial reasoning from fluid reasoning and in providing scores for the GAI in the standard print out, will actually better facilitate a more nuanced picture of the cognitive profiles associated with NF1 
and NF1+ADHD. Finally, we were unable to include additional questionnaire measures in the current study, which would have allowed for a greater understanding of the relation between comorbid ADHD and social, adaptive, behavioral, and academic functioning in children with NF1, and this is recommended for future research on this topic.

In summary, this study demonstrates that rather than a global downward shift in intellectual functioning, children with NF1 show an uneven intellectual profile with strengths in verbal reasoning and weaknesses in working memory and processing speed, supporting the inclusion of the GAI when evaluating cognitive functioning and the implementation of specific accommodations and interventions to address areas of cognitive deficit. In addition, this study found the children with NF1 and comorbid ADHD experience a "double hit" that negatively affects cognitive functioning, with the most intense burden in the area of working memory. These findings support the early diagnosis and treatment of ADHD in children with NF1, in order to improve cognitive outcomes.

Compliance with Ethical Standards All procedures followed were in accordance with the ethical standards of the responsible committee on human experimentation (institutional and national) and with the Helsinki Declaration of 1975, as revised in 2000 (5).

Conflict of Interest The authors declare that they have no competing interests.

Human and Animal Rights and Informed Consent No animals were involved in this research. This research project was approved by the Institutional Review Board (IRB) of Children National Medical Center, with appropriate informed consent procedures followed with all participants.

\section{References}

American Psychiatric Association (2000). Diagnostic and statistical manual of mental disorders (4th edn., text rev.). Washington, DC: Author.

American Psychiatric Association (2013). Diagnostic and statistical manual of mental disorders (5th edn.). Washington, DC: Author.

Barnea-Goraly, N., Menon, V., Eckert, M., Tamm, L., Bammer, R., Karchemskiy, A., Dant, C. C., \& Reizz, A. L. (2005). White matter development during childhood and adolescence: a cross-sectional diffusion tensor imaging study. Cerebral Cortex, 15(12), 18481854.

Benton, A. L., Hamsher, K., Varney, N. R., \& Spreen, O. (1983). Judgment of line orientation. New York: Oxford University Press.

Black, D. O., Wallace, G. L., Sokoloff, J. L., \& Kenworthy, L. (2009). Brief report: IQ split predicts social symptoms and communication abilities in high-functioning children with autism spectrum disorders. Journal of Autism and Developmental Disorders, 39(11), 1613-1619.

Bridgett, D., \& Walker, M. (2006). Intellectual functioning in adults with ADHD: a meta-analytic examination of full scale IQ differences between adults with and without ADHD. Psychological Assessment, 18(1), 1-14.

Cutting, L. E., Cooper, K. L., Koth, C. W., Mostofsky, S. H., Kates, W. R., Denckla, M. B., \& Kaufmann, W. E. (2002). Megalencephaly in NF1: predominantly white matter contribution and mitigation by ADHD. Neurology, 59(9), 1388-1394.

DuPaul, G. J., Power, T. J., Anastopoulos, A. D., \& Reid, R. (1998). ADHD rating scale - IV. New York: Guilford Press.

Ferner, R. E., Hughes, R. A. C., \& Weinman, J. (1996). Intellectual impairment in neurofibromatosis type 1. Journal of the Neurological Sciences, 138, 125-133.

Ferner, R. E., Huson, S. M., Thomas, N., Moss, C., Evans, D. G., Upadhyaya, M., Towers, R., Gleeson, M., Steiger, C., \& Kirby, A. (2007). Guidelines for the diagnosis and management of individuals with neurofibromatosis 1. Journal of Medical Genetics, 44(2), 8188.

Frazier, T., Demaree, H., \& Youngstrom, E. (2004). Meta-analysis of intellectual and neuropsychological test performance in attentiondeficit/hyperactivity disorder. Neuropsychology, 18(3), 543-555.

Froehlich, T. E., Lanphear, B. P., Epstein, J. N., Barbaresi, W. J., Katusic, S. K., \& Kahn, R. S. (2007). Prevalence, recognition, and treatment of attention-deficit/hyperactivity disorder in a national sample of US children. Archive of Pediatric Adolescent Medicine, 161(9), 857864.

Gutmann, D. H., Avlsworth, A., Carey, J. C., Korf, B., Marks, J., Pyeritz, R. E., Rubenstein, A., \& Viskochil, D. (1997). The diagnostic evaluation and multidisciplinary management of neurofibromatosis 1 and neurofibromatosis 2. Journal of American Medical Association, 278(1), 51-57.

Huson, S. M., Compston, D. A., Clark, P., \& Harper, P. S. (1989). A genetic study of von Recklinghausen neurofibromatosis in south east Wales: prevalence, fitness, mutation rate, and effect of parental transmission on severity. Journal of Medical Genetics, 26, 704-711.

Hyman, S. L., Shores, E. A., \& North, K. N. (2005). The nature and frequency of cognitive deficits in children with neurofibromatosis type 1. Neurology, 65, 1037-1044.

Hyman, S. L., Shores, E. A., \& North, K. N. (2006). Learning disabilities in children with neurofibromatosis type 1: subtypes, cognitive profiles, and attention-deficit-hyperactivity disorder. Developmental Medicine and Child Neurology, 48(12), 973-977.

Lawrence, K. E., Levitt, J. G., Loo, S. K., Ly, R., Yee, V., O’Neill, J., Alger, J., \& Narr, K. L. (2013). White matter microstructures in subjects with attention-deficit/hyperactivity disorder and their siblings. Journal of American Academy of Adolescent Psychiatry, 52(4), 431-440.

Levine, T. M., Materek, A., Abel, J., O’Donnell, M., \& Cutting, L. E. (2006). Cognitive profile of neurofibromatosis type 1. Seminar of Pediatric Neurology, 13(1), 8-20.

Lidzba, K., Granstrom, S., Lindenau, J., \& Mautner, V. F. (2012). The adverse influence of attention-deficit disorder with or without hyperactivity on cognition in neurofibromatosis type 1. Developmental Medicine and Child Neurology, 54, 892-897.

Mayes, S. D., \& Calhoun, S. L. (2006). WISC-IV and WISC-III profiles in children with ADHD. Journal of Attention Disorders, 9(3), 486493.

Moffitt, T. E., Caspi, A., Harkness, A. R., \& Silva, P. A. (1993). The natural history of change in intellectual performance: who changes? How much? Is it meaningful? Journal of Child Psychology and Psychiatry, 34(4), 455-506.

National Institutes of Health (1987). Neurofibromatosis: consensus statement. 6(12), 1-19.

North, K. N. (1998). Neurofibromatosis 1 in childhood. Seminars in Pediatric Neurology, 5(4), 231-242.

North, K. N. (2000). Neurofibromatosis 1. American Journal of Medical Genetics, 97, 119-127. 
North, K. N., Hyman, S. L., \& Barton, B. (2002). Cognitive defects in neurofibromatosis 1. Journal of Child Neurology, 17(8), 605-612.

Oliveras-Rentas, R. E., Kenworthy, L., Roberson, R., Martin, A., \& Wallace, G. L. (2011). WISC-IV profile in high-functioning autism spectrum disorders: impaired processing speed is associated with increased autism communication symptoms and decreased adaptive communication abilities. Journal of Autism and Developmental Disorders, 42, 655-664.

Payne, J. M., Hyman, S. L., Shores, E. A., \& North, K. N. (2011). Assessment of executive function and attention in children with neurofibromatosis type 1: relationships between cognitive measures and real-world behavior. Child Neuropsychology, 17(4), 313-329.

Payne, J. M., Arnold, S. S., Pride, N. A., \& North, K. N. (2012). Does attention-deficit-hyperactivity disorder exacerbate executive dysfunction in children with neurofibromatosis type 1 ? Developmental Medicine and Child Neurology, 54, 898-904.

Pride, N. A., Payne, J. M., \& North, K. N. (2012). The impact of ADHD on the cognitive and academic functioning of children with NF1. Developmental Neuropsychology, 37(7), 590-600.

Raiford, S.E.R., Weiss, L.G., Rolfhus, E., \& Coalson, D. (2005). GAI utility: introduction to the General Ability Index (GAI) utility. Pearson.

Rosser, T. L., \& Packer, R. J. (2003). Neurocognitive dysfunction in children with neurofibromatosis type 1. Current Neurology and Neuroscience Report, 3(2), 129-136.

Rowbotham, I., Cate, I. M., Sonuga-Barke, E. J. S., \& Huijbregts, S. C. J. (2009). Cognitive control in adolescents with neurofibromatosis type 1. Neuropsychology, 23(1), 50-60.

Roy, A., Roulin, J.-L., Charbonnier, V., Allain, P., Fasotti, L., Barbarot, S., Stalder, J.-F., Terrien, A., \& Le Gall, D. (2010). Executive dysfunction in children with neurofibromatosis type 1: a study of action planning. Journal of the International Neuropsychological Society, 16, 1056-1063.

Schrimsher, G. W., Billingsley, R. L., Slopis, J. M., \& Moore, B. D. (2003). Visual-spatial performance deficits in children with neurofibromatosis type-1. American Journal of Medicine Genetics, 120A, 326-330.

Templer, A. K., Titus, J. B., \& Guttman, D. H. (2012). A neuropsychological perspective on attention problems in neurofibromatosis type 1. Journal of Attention Disorders, 20(10), 1-8.

Thaler, N. S., Bello, D. T., \& Etcoff, L. M. (2012). WISC-IV profiles are associated with differences in symptomatology and outcomes in children with ADHD. Journal of Attention Disorders, 17(4), 291301.

Ullebo, A. K., Posserud, M. B., Heiervang, E., Obel, C., \& Gillberg, C. (2012). Prevalence of the ADHD phenotype in 7- to 9-year old children: effect of informant, gender, and non-participation. Social Psychiatry and Psychiatric Epidemiology, 47(5), 763769.

Wechsler, D. (2003). Wechsler intelligence scale for children (4th ed.). New York: Psychological Corporation.

Wechsler, D. (2014). Wechsler intelligence scale for children (5th ed.). Bloomington: Psychological Corporation.

Weinstein, M., Ben-Sira, L., Levy, Y., Zachor, D. A., Ben Itzhak, E., Artzi, M., Tarrasch, R., Eksteine, P. M., Hendler, T., \& Ben Bashat, D. (2011). Abnormal white matter integrity in young children with autism. Human Brain Mapping, 32(4), 534-543. 